\title{
THE LEBESGUE INTEGRAL AS A RIEMANN INTEGRAL
}

\author{
ENRIQUE A. GONZALEZ-VELASCO \\ Department of Mathematics \\ University of Lowell \\ Lowel1, Massachusetts 01854 U.S.A. \\ (Recelved November 21, 1985 and in revised form December 30, 1986)
}

ABSTRACT. The object of this paper is to develop a very direct theory of the Lebesgue integral that is easily accessible to any audience familiar with the Riemann integral.

KEY WORDS AND PHRASES. Lebesgue integration, Riemann integration. 1980 AMS SUBJECT CLASSIFICATION CODE. 2801.

1. INTRODUCTION.

The problem of making the theory of Lebeggue integration accessible to a wide audience, including students of mathematics, science and engineering, is not easily solved. And yet, the Lebesgue integral is an indispensable tool in many branches of mathematics and its applications, such as Fourier analysis and signal processing.

The inaccessibility of the theory is mainly due to the fact that the usual presentations, both those that are and those that are not based on measure theory, are lengthy and indirect and rely on a number of new basic concepts that must be assimilated first. For instance, in Royden [1] the general definition of the Lebesgue integral is preceded by definitions for simple functions, then for bounded functions over a set of finite measure and then for nonnegative functions, and in each particular case some amount of theory is elaborated. In Apostol [2] the general definition is preceded by definitions for step functions and then for upper functions, and again a certain amount of theory is developed in each particular case. Furthermore, these classic presentations represent a new start, with all knowledge of Riemann integration to be abandoned in favor of the new Lebesgue theory. To avoid this, McShane [3] has recently developed a unified theory of Riemann and Lebesgue integration that is, hopefully, elementary enough for presentation to a wide audience. While sharing McShane's concern and agreeing that he succeeds in constructing an appealing unified theory, this author believes that, regarding simplicity and accessibility, the answer lies elsewhere.

There is an entirely different approach to the definition of the Lebesgue integral, due to Mikusiński [4], which is very direct and does not need the previous development of either measure theory or the Riemann integral. It is based, instead, on a series representation of the function to be integrated, but, since this series 
representation need not be unique, it requires a somewhat lengthy proof of consistency. In addition, it is not readily apparent how the definition of the Lebesgue Integral in any of the approaches mentloned so far can be immediately used for the quick computation of simple integrals.

Our task 1s, then, to construct a simple theory that builds on concepts familiar to any audience and that is based on a very direct and general definition, capable of dealing with the computation of simple integrals. To this end, the best course of action is to define the Lebesgue integral as a (possibly improper) Riemann integral. This is not only brief but allows for complete generality at once and, moreover, we know how to compute Riemann integrals (there is no need to develop a theory of improper Riemann integrals as a prerequisite because, beyond the definition, all that is needed is the fact that such an integral is a linear and increasing function of the integrand). Such a theory is presented in Rey Pastor [5], but only for functions defined on a set of finite measure and his development makes use of the RiemannStieltjes integral. The main object of this paper is to reconstruct this theory from the beginning (with one minor modification) for functions defined over sets of arbitrary measure and, for additional simplicity, avoiding all use of the RiemannStieltjes integral. This has necessitated a complete set of new proofs and a collection of new auxiliary results (Propositions 2, 3 and 4 and Lemmas 3,4 and 5 below). In addition, we have included the Levi monotone convergence theorem and have adopted an entirely different approach to the Lebesgue dominated convergence theorem. Instead of basing its proof on the additivity of the integral (not an easy fact to establish), our approach highlights the role of uniform convergence. Lemmas 3 and 4 show that the contribution of the dominated convergence is just to enable the uniform convergence to do the job via Egorov's theorem and Proposition 4.

2. THE LEBESGUE MEASURE AND MEASURABLE FUNCTIONS.

For easy reference, and to specify how much Lebesgue measure theory is necessary for our presentation, we collect in this section those results that will be used in the rest of the article. For proofs see [1] or [6]. By a rectangle in $\mathbb{R}^{\mathrm{n}}$ we shall mean the product space of $n$ bounded intervals, open, closed, or neither. The volume of such a rectangle $R$, denoted by $v(R)$, is the product of the lengths of its component intervals.

DEFINITION 1. The outer measure of a set $E \subset \mathbb{R}^{n}$ is

$$
m(E) \stackrel{\text { def }}{=} \text { inf } \sum v\left(R_{i}\right)
$$

where the infimum is taken over all finite or countable collections of open rectangles $\left\{R_{1}\right\}$ such that $E \subset \cup R_{1}$.

It turns out that it is not always true that the outer measure of a finite union of disjoint sets is the sum of their outer measures. But this will always be true if we restrict ourselves to sets of the following type.

DEFINITION 2. A set $E \subset \mathbb{R}^{n}$ is called measurable if and only if for any set $S \subset \mathbb{R}^{n} m(S)=m(S \cap E)+m\left(S \cap E^{c}\right)$, where $E^{c}$ is the complement of $E$ in $\mathbb{R}^{n}$. If $E$ is measurable $\mathrm{m}(\mathrm{E})$ is called the Lebesgue measure of $E$. Notice that $\mathbb{R}^{\mathrm{n}}$ and the empty set are measurable with Lebesgue measures $\infty$ 
and zero respectively. It is also clear that a set is measurable if and only it its complement is measurable and that if $E$ and $F$ are measurable with $F \subset E$ then $m(F) \leq m(E)$.

THEOREM 1 .

(1) If $E$ is a finite or countable set then $m(E)=0$.

(2) If $\left\{E_{i}\right\}$ is a finite or countable collection of measurable sets then their union $U E_{i}$ is measurable and $m\left(U E_{i}\right) \leq \sum m\left(E_{i}\right)$. If, in addition, the $E_{i}$ are disjoint then equality holds.

(3) If $\left\{E_{1}\right\}$ is a finite or countable collection of measurable sets then their intersection $\cap E_{1}$ is measurable. If, in addition, $m\left(E_{1}\right)<\infty$ and $E_{1+1} \subset E_{1}$ for all $i$ then $m\left(E_{i}\right) \rightarrow m\left(\cap E_{i}\right)$ as $i \rightarrow \infty$.

(4) If $E$ and $F$ are measurable then $E-F \stackrel{i e f}{=}\{x \in E: x \notin F\}$ is measurable. If, in addition, $m(E)<\infty$ and $F \subset E$ then $m(E-F)=m(E)-m(F)$.

(5) Every half space in $\mathbb{R}^{n}$ is measurable.

(6) Every rectangle in $\mathbb{R}^{n}$ is measurable and its Lebesgue measure is its volume.

(7) Open and closed sets are measurable.

DEFINITION 3. A function $\mathrm{f}: \mathrm{E} \subset \mathbb{R}^{\mathrm{n}} \rightarrow \mathbb{R}$ is measurable if and only if $E$ is measurable and for any $y \in \mathbb{R}$ the set $\{x \in E: f(x)>y\}$ is measurable.

THEOREM 2. The following statements are equivalent:

(1) For each $y \in \mathbb{R}$ the set $\{x \in E: f(x)>y\}$ is measurable.

(2) For each $y \in \mathbb{R}$ the set $\{x \in E: f(x) \geq y\}$ is measurable.

(3) For each $y \in \mathbb{R}$ the set $\{x \in E: f(x)<y\}$ is measurable.

(4) For each $y \in \mathbb{R}$ the set $\{x \in E: f(x) \leq y\}$ is measurable.

EXERCISE 1. If $E$ is measurable and $f: E \rightarrow \mathbb{R}$ is continuous then $f$ is measurable.

THEOREM 3. Let $E \subset \mathbb{R}^{\mathrm{n}}$ be a measurable set.

(1) If $f, g: E \rightarrow \mathbb{R}$ are measurable then $f+g$ is measurable.

(2) If $\left\{\mathrm{f}_{\mathrm{N}}\right\}$ is a sequence of measurable functions on $E$ and if $\mathrm{f}_{\mathrm{N}} \rightarrow \mathrm{f}$ on $E$ then $f$ is measurable.

THEOREM 4. (Egorov's theorem) Let $E \subset \mathbb{R}^{\mathrm{n}}$ be a measurable set and let $\left\{\mathrm{f}_{\mathrm{N}}\right\}$ be a sequence of measurable functions on $E$ such that $f_{N} \rightarrow f$ on $E$. For any $\delta>$ 0 there is a subset $G$ of $E$ with $m(G)<\delta$ and such that $f_{N} \rightarrow f$ uniformly on $E-G$.

3. THE LEBESGUE INTEGRAL.

The Lebesgue approach to define the integral of a function $f: E \subset \mathbb{R}^{n} \rightarrow \mathbb{R}$ is to partition its range, not its domain as in the Riemann theory. To be specific, consider the case of a measurable set $E$ with $m(E)<\infty$ and a measurable function $\mathrm{f}: \mathrm{E} \rightarrow \mathbb{R}$ whose values are between zero and $M>0$. If $0=\mathrm{y}_{0}<\mathrm{y}_{1}<\ldots<\mathrm{y}_{\mathrm{k}}=\mathrm{M}$ is a partition of $[0, M]$ and if we define $s_{i}=\left\{x \in E: f(x)>y_{i}\right\}$ for $i=0$, $\ldots, k$, then $f$ is said to be integrable on $E$ if and only if

$$
\sup \sum_{i=1}^{k} m\left(s_{i}\right)\left(y_{i}-y_{i-1}\right)=\inf \sum_{i=1}^{k} m\left(s_{i-1}\right)\left(y_{i}-y_{i-1}\right)
$$

where the supremum and infimum are taken over all partitions of $[0, M]$, and this 
common value will be denoted by $\int_{E} f$.

EXERCISE 2. Define a set $E_{i}=\left\{x \in E: y_{i-1}<f(x) \leq y_{i}\right\}$ for each i. Prove that

$$
\sum_{i=1}^{k} m\left(E_{i}\right) y_{i-1}=\sum_{i=1}^{k} m\left(S_{i}\right)\left(y_{i}-y_{i-1}\right)
$$

and

$$
\sum_{i=1}^{k} m\left(E_{i}\right) y_{i}=\sum_{i=1}^{k} m\left(S_{i-1}\right)\left(y_{i}-y_{i-1}\right) \text {. }
$$

Now, if we define a function $\mu_{f}:[0, M] \rightarrow \mathbb{R}$ by $\mu_{f}(y)=m\{x \in E: f(x)>y\}$ then $\mu_{f}$ is clearly bounded and decreasing and, thus, Riemann integrable on $[0, M]$. PROPOSITION 1. Let $E \subset \mathbb{R}^{\mathrm{n}}$ be a measurable set with $m(E)<\infty$. Every bounded, nonnegative measurable function $f: E \rightarrow \mathbb{R}$ is integrable on $E$ and

$$
\int_{E} f=R \int_{0}^{M} \mu_{f},
$$

where the $R$ indicates Riemann integral.

PROOF. Notice that $m\left(S_{i}\right)=\mu_{f}\left(y_{i}\right)$ and then, since $\mu_{f}$ is decreasing,

$$
\sum_{i=1}^{k} m\left(s_{1}\right)\left(y_{1}-y_{i-1}\right) \quad \text { and } \quad \sum_{i=1}^{k} m\left(s_{i-1}\right)\left(y_{i}-y_{i-1}\right)
$$

are, respectively, the lower and upper Riemann sums of $\mu_{f}$ with respect to the partition $0=y_{0}<y_{1}<\cdots<y_{k}=M$ of $[0, M]$. The result follows because $\mu_{f}$ is Riemann integrable on $[0, M]$, Q.E.D.

If $f$ is nonpositive instead of nonnegative we would like its integral on $E$ to equal the opposite of the integral of $-f$, that is, we want

$$
\int_{E} f=-R \int_{0}^{M} \mu_{-f}=R \int_{M}^{0} \mu_{-f}=R \int_{-M}^{0}-\mu_{-f}(-y) d y .
$$

This suggests that we depine $\mu_{f}(y)=-\mu_{-f}(-y)$ if $y<0$, and then the entire preceding discussion motivates the following general definitions.

DEFINITION 4. Let $E \subset \mathbb{R}^{n}$ be a measurable set and let $\mathrm{f}: \mathrm{E} \rightarrow \mathbb{R}$ be a measurable function. The measure function of $f$ on $E$ is the function $\mu_{f}$ defined by

$$
\mu_{f}(y)=\left\{\begin{aligned}
m\{x \in E: f(x)>y\} & \text { if } y>0 \\
-m\{x \in E: f(x)<y\} & \text { if } y<0
\end{aligned}\right.
$$

Notice that $\mu_{\mathrm{f}} \leq 0$ on $(-\infty, 0)$ and $\mu_{\mathrm{f}} \geq 0$ on $(0, \infty)$, and that $\mathrm{y}_{1} \leq \mathrm{y}_{2} \Rightarrow$ $\mu_{\mathrm{f}}\left(\mathrm{y}_{1}\right) \geq \mu_{\mathrm{f}}\left(\mathrm{y}_{2}\right)$ on each of these intervals. The measure function can have infinite values if $m(E)=\infty$, but, if it does not, it is Riemann integrable on every bounded subinterval of $(-\infty, 0)$ or $(0, \infty)$, and then the improper integrals

$$
R \int_{-\infty}^{0} \mu_{\mathrm{f}} \quad \text { and } \quad R \int_{0}^{\infty} \mu_{\mathrm{f}}
$$

exist (they may be infinite). For convenience, if $\mu_{f}$ has infinite values on $(-\infty, 0)$ or on $(0, \infty)$ we define

$$
R \int_{-\infty}^{0} \mu_{\mathrm{f}}=-\infty \quad \text { or } \quad R \int_{0}^{\infty} \mu_{\mathrm{f}}=\infty \text {. }
$$


DEFINITION 5. Let $E \subset \mathbb{R}^{n}$ be a measurable set, $f: E \rightarrow \mathbb{R}$ a measurable function, and $\mu_{f}$ the measure function of $f$ on $E$. Then the (Lebesgue) integral of $f$ on $E$ is defined to be

$$
\int_{E} f=R \int_{-\infty}^{\infty} \mu_{f}
$$

if the right-hand side exists (that is if the two improper Riemann integrals over $(-\infty, 0)$ and $(0, \infty)$ exist). If, in addition, the right-hand side is finite then $f$ is said to be (Lebesgue) integrable on $E$ and we write $\rho$ e $L(E)$.

EXAMPLE 1. If $E=\{x \in \mathbb{R}: 0<x<1\}$ and $f(x)=1 / \sqrt{x}$ we have $\mu_{f}(y)=0$ if $\mathrm{y}<0, \mu_{\mathrm{f}}(\mathrm{y})=1$ if $0<\mathrm{y}<1$ and $\mu_{\mathrm{f}}(\mathrm{y})=\mathrm{y}^{-2}$ if $\mathrm{y} \geq 1$, and then

$$
\int_{E} \mathbf{f}=R \int_{0}^{1} d y+R \int_{1}^{\infty} y^{-2} d y=2 \text {. }
$$

EXAMPLE 2. If $E=\{x \in \mathbb{R}: 0<\mathrm{x}<1\}$ and $\mathrm{f}(\mathrm{x})=1 / \mathrm{x}$ then $\mu_{\mathrm{f}}(\mathrm{y})=0$ if $y<0, \quad \mu_{f}(y)=1$ if $0<y<1$ and $\mu_{f}(y)=1 / y$ if $y \geq 1$. We have

$$
\int_{E} P=R \int_{0}^{1} d y+R \int_{1}^{\infty} \frac{1}{y} d y=\infty \text {. }
$$

Examples 1 and 2 show that the product of integrable functions need not be integrable.

EXAMPLE 3. If $E=\{x \in \mathbb{R}: 0<x<1\}$ and $f(x)=1$ when $x$ is rational and $f(x)=0$ when $x$ is irrational, then $\mu_{f}(y)=0$ for all $y \neq 0$ and $\int_{E} f=0$.

EXERCISE 3. Show that if $f \geq 0$ and $\int_{E} f=0$ then $m\{x \in E: f(x) \neq 0\}=0$. 4. BASIC PROPERTIES OF THE LEBESGUE INTEGRAL.

First we establish the basic properties of the measure function. We shall write $\mu_{f}^{E}$ instead of $\mu_{f}$ when we need to specify the domain $E$ of $f$.

LEMMA 1. Let $E \subset \mathbb{R}^{n}$ be a measurable set, let $f, g: E \rightarrow \mathbb{R}$ be measurable functions and let $c \in \mathbb{R}$. Then

(1) $f \leq g \Rightarrow \mu_{\mathrm{f}} \leq \mu_{\mathrm{g}}$.

(2) cf is measurable and $\mu_{\mathrm{cf}}(\mathrm{y})=\operatorname{sgn}(\mathrm{c}) \mu_{\mathrm{f}}(\mathrm{y} / \mathrm{c})$ for all $\mathrm{y} \neq 0$. PROOF.

(1) If $y>0,\{x \in E: f(x)>y\} \subset\{x \in E: g(x)>y\} \Rightarrow \mu_{f}(y) \leq \mu_{g}(y)$. If $y<0, \quad\{x \in E: g(x)<y\} \subset\{x \in E: f(x)<y\} \Rightarrow-\mu_{g}(y) \leq-\mu_{f}(y) \Rightarrow \mu_{f}(y) \leq \mu_{g}(y)$.

(2) We shall consider the case $c<0, y>0$, and the others are similar.

$$
\begin{aligned}
\mu_{c f}(y) & =m\{x \in E: c f(x)>y\} \\
& =m\{x \in E: f(x)<y / c\} \\
& =-\mu_{f}(y / c) \\
& =\operatorname{sgn}(c) \mu_{f}(y / c)
\end{aligned}
$$

Q.E.D.

LEMMA 2. If $\left\{E_{i}\right\}$ is a finite or countable collection of disjoint measurable sets in $\mathbb{R}^{n}$, if $E=U E_{i}$ and if $f: E \rightarrow \mathbb{R}$ is a measurable function, then $\mu_{f}^{E}=$ $\sum \mu_{f}^{E_{i}}$.

PROOF. If $y>0, \mu_{f}^{E}(y)=m\{x \in E: f(x)>y\}=m\left(U\left\{x \in E_{i}: f(x)>y\right\}\right)=$ $\sum m\left\{x \in E_{i}: f(x)>y\right\}=\sum \mu_{f}^{E}$. Similarly if $y<0$, Q.E.D. 
COROLLARY 1. If $E, F \subset \mathbb{R}^{n}$ are measurable sets with $F \subset E$ and if $f: E \rightarrow \mathbb{R}$ is a measurable function, then $\mu_{f}^{F} \leq \mu_{f}^{E}$ on $(0, \infty)$ and $\mu_{f}^{F} \geq \mu_{f}^{E}$ on $(-\infty, 0)$.

PROOF. $\quad E=F \cup(E-F) \Rightarrow \mu_{f}^{E}=\mu_{f}^{F}+\mu_{f}^{E-F}$. Then $\mu_{f}^{E-F} \geq 0$ on $(0, \infty) \Rightarrow \mu_{f}^{F} \leq$ $\mu_{f}^{E}$ on $(0, \infty)$ and $\mu_{f}^{E-F} \leq 0$ on $(-\infty, 0) \Rightarrow \mu_{f}^{F} \geq \mu_{f}^{E}$ on $(-\infty, 0)$, Q.E.D.

THEOREM 5. Let $E, F \subset \mathbb{R}^{n}$ be measurable sets, let $f, g: E \rightarrow \mathbb{R}$ be measurable functions and let $c e \mathbb{R}$. Then, if the integrals below exist,

(1) $f \leq 8 \Rightarrow \int_{E} f \leq \int_{E} g$ and if, in addition, $f \geq 0$ then $g \in L(E) \Rightarrow$ $f \in L(E)$.

(2) $\int_{E} c f=c \int_{E} f$ and then $f \in L(E) \Rightarrow$ cf $\in L(E)$.

(3) If $F \subset E$ then $f \in L(E) \Rightarrow f \in L(F)$ and if, in addition, $f \geq 0$ then $\int_{F} f \leq \int_{E} f$

(4) If $c>0$ and if $f^{c} \stackrel{\operatorname{def}}{=} \min \{f, c\}$ then $\int_{E} f^{c} \rightarrow \int_{E} f$ as $c \rightarrow \infty$.

PROOF. (1) is obvious from Lemma 1(1).

(2) Assume that $c<0$ (proceed analogously if $c>0$ ). By Lemma $1(2)$,

$$
R \int_{0}^{b} \mu_{c f}(y) d y=R \int_{0}^{b}-\mu_{f}(y / c) d y=c R \int_{b / c}^{0} \mu_{f}(u) d u
$$

and taking the limit as $b \rightarrow \infty$,

$$
R \int_{0}^{\infty} \mu_{c f}=c R \int_{-\infty}^{0} \mu_{f}
$$

Similarly for the integral from $-\infty$ to 0 . Adding the two proves (2).

(3) By Corollary 1,

$$
R \int_{-\infty}^{0} \mu_{f}^{E} \leq R \int_{-\infty}^{0} \mu_{f}^{F} \leq 0 \leq R \int_{0}^{\infty} \mu_{f}^{F} \leq R \int_{0}^{\infty} \mu_{f}^{E}
$$

If the outer integrals are finite, so are the inner integrals. Thus, $f \in L(E) \Rightarrow$ $f \in L(E)$. The last assertion follows from the last two inequalities on the right.

$$
\int_{E} f^{c}=R \int_{-\infty}^{c} \mu_{f} c=R \int_{-\infty}^{c} \mu_{f} \rightarrow R \int_{-\infty}^{\infty} \mu_{f}=\int_{E} f
$$

as $\quad \mathrm{C} \rightarrow \infty$, Q.E.D.

THEOREM 6. Let $E_{1}, \ldots, E_{N}$ be a finite collection of disjoint measurable sets in $\mathbb{R}^{n}$, let $E=U E_{1}$ and let $f: E \rightarrow \mathbb{R}$ be a measurable function. Then

$$
\int_{E} f=\sum \int_{E_{i}} f \text {. }
$$

Furthermore, if $f$ has a constant value $c_{i}$ on each $E_{i}$, then

$$
\int_{E} f=\sum c_{i} m\left(E_{i}\right)
$$

PROOF. The first assertion follows from Lemma 2 and Definition 5 . Now, if $c_{i}>$

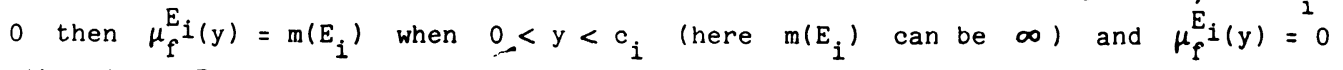
otherwise. Then

$$
\int_{E_{i}} f=R \int_{0}^{c_{i}} \mu_{f}^{E_{i}}=c_{i} m\left(E_{i}\right) .
$$

Similarly if $c_{i}<0$, Q.E.D. 
EXERCISE 4. If there are constants $a, b \in \mathbb{R}$ such that $a \leq f(x) \leq b$ for all $x \in E$, then $a m(E) \leq I_{E} f \leq b m(E)$ and $m(E)<\infty \Rightarrow f \in L(E)$.

EXERCISE 5. Let $E \subset \mathbb{R}^{n}$ be a measurable set and let $f, g: E \rightarrow \mathbb{R}$ be measurable functions. If $f=g$ on $E$ except, possibly, on a subset of $E$ of measure zero, then $\int_{E} \mathbf{f}=\int_{E} \mathbf{8}$.

THEOREM 7. Let $E \subset \mathbb{R}^{n}$ be a measurable set and let $f: E \rightarrow \mathbb{R}$ be a measurable function. If $\int_{E} f$ exists, then

$$
\left|\int_{E} f\right| \leq \int_{E}|f|
$$

Also, $f \in L(E) \Rightarrow|f| \in L(E)$.

PROOF. If $y>0$ the identity $\{x \in E:|f(x)|>y\}=\{x \in E: f(x)>y\} U$ $\{x \in E: f(x)<-y\}$ shows that $|f|$ is measurable and that $\mu_{|f|}(y)=\mu_{f}(y)-\mu_{f}(-y)$. Now,

$$
R \int_{0}^{\infty} \mu_{\mathrm{f}}(-\mathrm{y}) \mathrm{dy}=-R \int_{0}^{-\infty} \mu_{\mathrm{f}}(u) d u=R \int_{-\infty}^{0} \mu_{\mathrm{f}}(\mathrm{y}) \mathrm{dy},
$$

so that

$$
R \int_{0}^{\infty} \mu_{|f|}=R \int_{0}^{\infty}\left(\mu_{\mathrm{f}}(y)-\mu_{\mathrm{f}}(-\mathrm{y})\right) d y=R \int_{0}^{\infty} \mu_{\mathrm{f}}-R \int_{-\infty}^{0} \mu_{\mathrm{f}} .
$$

The right-hand side is always well defined as a real number or $\infty$ since $\mu_{\mathrm{f}} \leq 0$ on $(-\infty, 0)$ and $\mu_{f} \geq 0$ on $(0, \infty)$, and it is fini,te if and only if both integrals are finite, that is $f \in L(E) \Rightarrow|f| \in L(E)$. In either case, the inequality $|a+b| \leq$ $a-b$ holds when $a \geq 0$ and $b \leq 0$, when $a \geq 0$ and $b=-\infty$, and when $a=\infty$ and $b \leq 0$. Thus,

$$
\left|\int_{E} f\right|=\left|R \int_{0}^{\infty} \mu_{f}+R \int_{-\infty}^{0} \mu_{f}\right| \leq \int_{E}|f|
$$

Q.E.D.

EXERCISE 6. Let $R$ be a rectangle in $\mathbb{R}^{n}$. If $f: R \rightarrow \mathbb{R}$ is Riemann integrable then $f \in L(E)$ and $\int_{E} f=R \int_{E} f$.

5. THE ADDITIVITY OF THE LEBESGUE INTEGRAL.

This is one of the week points of the Lebesgue theory and it will not be straightforward to establish this fact. We start by considering two particular cases: those of piecewise continuous functions and nonnegative bounded functions.

PROPOSITION 2. Let $E \subset \mathbb{R}^{n}$ be a measurable set and let $f, g: E \rightarrow \mathbb{R}$ be measurable functions each of which has a finite number of values. Then

$$
\int_{E}(f+g)=\int_{E} f+\int_{E} g
$$

PROOF. If $f$ has only a finite number of values $c_{1}, \ldots, c_{N}$ on disjoint subsets $F_{1}, \ldots, F_{N}$ of $E$ and if $g$ has only a finite number of values $C_{1}, \ldots, C_{K}$ on disjoint subsets $G_{1}, \ldots, G_{K}$ of $E$, then $f+g$ is the function whose values are $c_{i}+c_{j}$ on $F_{i} \cap G_{j}$. Noticing that $F_{i}$ is the disjoint union $\sum_{j=1} F_{i} \cap G_{j}$ and using Theorem 6 we have

$$
\int_{E}(f+g)=\sum \underset{i=1}{N} \sum_{j=1}^{K}\left(c_{i}+c_{j}\right) m\left(F_{i} \cap G_{j}\right)
$$




$$
\begin{aligned}
& =\sum_{i=1}^{N} c_{1} \sum_{j=1}^{K} m\left(F_{i} \cap G_{j}\right)+\sum_{j=1}^{K} c_{j} \sum_{i=1}^{N} m\left(G_{j} \cap F_{i}\right) \\
& =\sum_{i=1}^{N} c_{i} m\left(F_{i}\right)+\sum_{j=1}^{K} c_{j} m\left(G_{j}\right) \\
& =\int_{E} f+\int_{E} B
\end{aligned}
$$

Q.E.D.

PROPOSITION 3. Let $E \subset \mathbb{R}^{n}$ be a measurable set and let $f, g: E \rightarrow \mathbb{R}$ be nonnegative, bounded, measurable functions. Then

$$
\int_{E}(f+g)=\int_{E} f+\int_{E} g
$$

PROOF. Let $f$ have values in $[0, M], M>0$, and let $0=y_{0}<y_{1}<\ldots<y_{k}$ $=M$ be a partition of $[0, M]$. Then define $F_{i}=\left\{x \in E: f(x)>y_{i}\right\}$ and a function $s_{f} \leq f$ by $s_{f}(x)=y_{i-1}$ if $x \in F_{i-1}-F_{i}, i=1, \ldots, k$. Noticing that $y_{0}$ $=m\left(F_{k}\right)=0$ and using Theorem 6 we have

$$
\begin{aligned}
\int_{E} s_{f} & =\sum_{i=1}^{k} y_{i-1} m\left(F_{i-1}-F_{i}\right) \\
& =\sum_{i=1}^{k} y_{i-1}\left(m\left(F_{i-1}\right)-m\left(F_{i}\right)\right) \\
& =\sum_{i=1}^{k}\left(y_{i}-y_{i-1}\right) m\left(F_{i}\right) \\
& =\sum_{i=1}^{k} \mu_{f}\left(y_{i}\right)\left(y_{i}-y_{i-1}\right) \\
& \leq \int_{E} f,
\end{aligned}
$$

and, as the norm of the partition approaches zero,

$$
\int_{E} s_{f} \rightarrow R \int_{0}^{M} \mu_{f}=\int_{E} f
$$

If $s_{g}$ is defined for $g$ as $s_{f}$ was defined for $f$, applying Proposition 2 to $s_{f}$ and $s_{g}$, observing that $s_{f}+s_{g} \leq f+g$, using Theorem $5(1)$, and letting the norm of the partition approach zero, we obtain

$$
\int_{E}(f+g) \geq \int_{E}\left(s_{f}+s_{g}\right)=\int_{E} s_{f}+\int_{E} s_{g} \rightarrow \int_{E} f+\int_{E} g \text {. }
$$

The first integral above is well defined because $f+g$ is measurable, nonnegative and bounded. Similarly, if we define a function $S_{f} \geq f$ by $S_{f}(x)=y_{i}$ if $x \in$ $F_{i-1}-F_{i}$ and if $S_{g}$ is defined for $g$ as $S_{f}$ is for $f$, a similar argument would yield

$$
\int_{E}(f+g) \leq \int_{E} f+\int_{E} g
$$

and the conclusion follows from the last two inequalities, Q.E.D.

THEOREM 8. Let $E \subset \mathbb{R}^{n}$ be a measurable set and let $f, g \in L(E)$. Then $f+g$ $\epsilon L(E)$ and

$$
\int_{E}(f+g)=\int_{E} f+\int_{E} g
$$


PROOF. Assume first that $f$ and $g$ are nonnegative and for $M>0$ define $f^{M}$ $=\min \{f, M\}$ and, similarly, $g^{M}$ and $(f+g)^{M}$. Since $f^{M}+g^{M} \leq f+g$, Proposition 3 gives

$$
\int_{E} f^{M}+\int_{E} g^{M}=\int_{E}\left(f^{M}+g^{M}\right) \leq \int_{E}(f+g)
$$

and then Theorem 5(4) implies that

$$
\int_{E} f^{M}+\int_{E} g^{M} \rightarrow \int_{E} f+\int_{E} g \leq \int_{E}(f+g) .
$$

as $M \rightarrow \infty$. It is not difficult to see that $(f+g)^{M} \leq f^{M}+g^{M}$, and then

$$
\int_{E}(f+g)^{M} \leq \int_{E}\left(f^{M}+g^{M}\right)=\int_{E} f^{M}+\int_{E} g^{M} \leq \int_{E} f+\int_{E} g .
$$

Hence

$$
\int_{E}(f+g)^{M} \rightarrow \int_{E}(f+g) \leq \int_{E} f+\int_{E} g
$$

as $M \rightarrow \infty$. It follows that

$$
\int_{E}(f+g)=\int_{E} f+\int_{E} g
$$

and, since the right-hand side is finite, $f+g \in L(E)$.

If, instead, $f \geq 0$ and $g \leq 0$, define $E^{+}=\{x \in E: f+g \geq 0\}$ and $E^{-}=$ $\{x \in E: f+g<0\}$. Since $f+g,-g$ and $f=f+g+(-g)$ are nonnegative on $\mathrm{E}^{+}$, the preceding proof shows that

$$
\int_{E^{+}} f=\int_{E^{+}}(f+g)+\int_{E^{+}}(-g)=\int_{E^{+}}(f+g)-\int_{E^{+}} g \text {. }
$$

Similarly, $-(f+g), \quad f$ and $-g=-(f+g)+f$ are nonnegative on $E^{-}$and

$$
\int_{E^{-}}(-g)=-\int_{E^{-}}(f+g)+\int_{E^{-f}}
$$

Subtracting the last equation from the previous one and using Theorem 6 proves the desired result in this case.

The two cases proved above imply the truth of the assertion in the general case after decomposing $E$ into the union of four disjoint subsets, on each of which $f$ and $g$ do not change sign, Q.E.D.

6. THE CONVERGENCE THEOREMS.

In this section we study the validity of the limit $\int_{E} f_{N} \rightarrow \int_{E} f$ when $f_{N} \rightarrow f$. In the case of Riemann integration the uniform convergence of this sequence is a sufficient condition. We start by proving a similar result for the Lebesgue integral. PROPOSITION 4. Let $S \subset \mathbb{R}^{n}$ be a measurable set with $m(S)<\infty$ and let $\left\{\mathrm{f}_{N}\right\}$ be a sequence of measurable functions such that $f_{N} \in L(S)$ and $f_{N} \rightarrow f \in L(S)$ uniformly on $S$. Then

$$
\int_{S} e_{N} \rightarrow \int_{S} f
$$

PROOF. Given $\epsilon>0$ there is a $K \in \mathbb{Z}^{+}$such that $f-\epsilon \leq f_{N} \leq f+\epsilon$, and 


$$
\text { then } \begin{aligned}
\mu_{\mathrm{f}-\epsilon} \leq \mu_{\mathrm{f}_{\mathrm{N}}} \leq \mu_{\mathrm{f}+\epsilon}, \text { for } \mathrm{N}>\mathrm{K} & \text {. } \\
\text { For } \mathrm{y}>0 \text { and } \mathrm{N}>\mathrm{K}, & \\
\mu_{\mathrm{f}}(\mathrm{y}+\epsilon) & =\mathrm{m}\{\mathrm{x} \epsilon \mathrm{S}: \mathrm{f}(\mathrm{x})>\mathrm{y}+\epsilon\} \\
& =\mathrm{m}\{\mathrm{x} \epsilon \mathrm{S}: \mathrm{f}(\mathrm{x})-\epsilon>\mathrm{y}\} \\
& =\mu_{\mathrm{f}-\epsilon}(\mathrm{y}) \\
& \leq \mu_{\mathrm{f}_{\mathrm{N}}}(\mathrm{y}),
\end{aligned}
$$

and then

$$
R \int_{0}^{\infty} \mu_{f}-\epsilon m(S) \leq R \int_{\epsilon}^{\infty} \mu_{f}=R \int_{0}^{\infty} \mu_{f}(y+\epsilon) d y \leq R \int_{0}^{\infty} \mu_{f_{N}} .
$$

For $\mathrm{y}>\epsilon$ and $\mathrm{N}>\mathrm{K}$,

$$
\begin{aligned}
\mu_{f_{N}}(y) & \leq \mu_{f+\epsilon}(y) \\
& =m\{x \in S: f(x)+\epsilon>y\} \\
& =m\{x \in S: f(x)>y-\epsilon\} \\
& =\mu_{f}(y-\epsilon)
\end{aligned}
$$

and then

$$
\begin{aligned}
R \int_{0}^{\infty} \mu_{\mathrm{f}_{\mathrm{N}}} & \leq \epsilon \mathrm{m}(\mathrm{S})+R \int_{\epsilon}^{\infty} \mu_{\mathrm{f}_{\mathrm{N}}} \\
& \leq \epsilon \mathrm{m}(\mathrm{S})+R \int_{\epsilon}^{\infty} \mu_{\mathrm{f}}(\mathrm{y}-\epsilon) \mathrm{dy} \\
& =\epsilon \mathrm{m}(\mathrm{S})+R \int_{0}^{\infty} \mu_{\mathrm{f}} .
\end{aligned}
$$

Thus, given $\epsilon>0$ there is a $K \in \mathbb{Z}^{+}$such that

$$
-\epsilon m(S) \leq R \int_{0}^{\infty} \mu_{f_{N}}-R \int_{0}^{\infty} \mu_{\mathrm{f}} \leq \epsilon \mathrm{m}(\mathrm{S})
$$

for $N>K$ and, since $\epsilon$ is arbitrary,

$$
R \int_{0}^{\infty} \mu_{\mathrm{f}_{\mathrm{N}}} \rightarrow R \int_{0}^{\infty} \mu_{\mathrm{f}}
$$

as $\mathrm{N} \rightarrow \infty$. Similarly,

$$
R \int_{-\infty}^{0} \mu_{\mathrm{f}_{\mathrm{N}}} \rightarrow R \int_{-\infty}^{0} \mu_{\mathrm{f}}
$$

as $\mathbf{N} \rightarrow \infty$. The last two limits and Definition 5 establish the desired convergence, Q.E.D.

The following theorem is usually cited as one of the triumphs of the Lebesgue theory because it replaces the uniform convergence of $\left\{\mathrm{f}_{\mathrm{N}}\right\}$ by the less restrictive condition that the $\mathrm{f}_{\mathrm{N}}$ be uniformly bounded. It also allows the domain of these functions to have arbitrary Lebesgue measure.

THEOREM 9. (The Lebesgue dominated convergence theorem) Let $E \subset \mathbb{R}^{n}$ be a measurable set and let $\left\{\mathrm{f}_{\mathrm{N}}\right\}$ be a sequence of integrable functions on $E$ that converges to an integrable function $f$ on $E$. If there is a function $g \in L(E)$ such 
that $\left|f_{N}\right| \leq g$ for all $N \in \mathbb{Z}^{+}$, then

$$
\int_{E} f_{N} \rightarrow \int_{E} f
$$

as $\mathrm{N} \rightarrow \infty$.

This theorem is sometimes stated with the $f_{N}$ and $f$ measurable rather than integrable, but, in fact, these functions must be integrable. For the $f_{N}$ this is so because $g \in L(E) \Rightarrow-g \in L(E)$ and $-g \leq f_{. N} \leq g \Rightarrow \mu_{-g} \leq \mu_{f_{N}} \leq \mu_{g}$. For $f$ because $\mathrm{f}_{\mathrm{N}} \rightarrow \mathrm{f}$ and $-\mathrm{g} \leq \mathrm{f}_{\mathrm{N}} \leq \mathrm{g} \Rightarrow-\mathrm{g} \leq \mathrm{f} \leq \mathrm{g}$.

The mechanism through which the uniform boundedness by the integrable function $g$ operates in this theorem can be better understood by means of the next two lemmas, whose proof is temporarily postponed.

LEMMA 3. Let $E \subset \mathbb{R}^{n}$ be a measurable set and let $g \in L(E)$. For any $\epsilon>0$ there is a subset $F$ of $E$ with $m(F)<\infty$ such that

$$
\left|\int_{E-F} g\right|<\epsilon
$$

LEMMA 4. Let $F \subset \mathbb{R}^{n}$ be a measurable set, let $g e L(F)$ and let $G$ be a subset of $F$. For any $e>0$ there is a $\delta>0$ such that if $m(G)<\delta$ then

$$
\left|\int_{G} g\right|<\epsilon
$$

Because of Lemma 3, the integrals of all functions involved in Theorem 9 are negligeable outside a set $F$ of finite measure and, because of Lemma 4 , the same is true in a sufficiently small subset $G$ of $F$. This is significant because, according to Egorov's theorem (Theorem 4), the set $G$ can be chosen so that $f_{N} \rightarrow f$ uniformly on $F-G$. And now, as in the case of Riemann integration, uniform convergence is seen to be the main driving force in the proof of Theorem 9 through Proposition 4.

PROOF OF THEOREM 9. Given $\epsilon>0$ let $F$ be as in Lemma 3 , let $\delta$ be as in Lemma 4 and, for this choice of $\delta$, let $G$ be as in Egorov's theorem. Using the triangle inequality and Theorems 6 and 7 we obtain

$$
\begin{aligned}
\left|\int_{E} f_{N}-\int_{E} p\right| & \leq\left|\int_{F} f_{N}-\int_{F} f\right|+\int_{E-F}\left|f_{N}\right|+\int_{E-F}|f| \\
& \leq\left|\int_{F} f_{N}-\int_{F} f\right|+2 \int_{E-F} g \\
& \leq\left|\int_{F-G} f_{N}-\int_{F-G} f\right|+\int_{G}\left|f f_{N}\right|+\int_{G}|f|+2 \int_{E-F} g \\
& \leq\left|\int_{F-G} f_{N}-\int_{F-G} f\right|+2 \int_{G} g+2 \int_{E-F} g \\
& \leq\left|\int_{F-G} f_{N}-\int_{F-G} f\right|+4 \epsilon .
\end{aligned}
$$

By Proposition 4, $N$ can be chosen so large that the first term on the right is smaller than $\epsilon$, and, since $\epsilon$ is arbitrary, the result follows, Q.E.D.

PROOF OF LEMMA 3. In view of Theorem 7 we need only consider the case $g \geq 0$. By Definition 5, given $\epsilon>0$ there is an $a>0$ such that

$$
R \int_{0}^{\mathrm{a}} \mu_{\mathrm{g}}^{\mathrm{E}}<\epsilon
$$


If we define $F=\{x \in E: g(x)>a\}$ we have $m(F)=\mu_{g}^{E}(a)<\infty$ since $g \in L(E)$. But $E-F=\{x \in E: 0 \leq B(x) \leq a\}$, so that $\mu_{g}^{E-F}(y) \leq \mu_{g}^{E}(y)$ if $y<a$ and $\mu_{\mathrm{g}}^{\mathrm{E}-\mathrm{F}}(\mathrm{y})=0$ if $\mathrm{y} \geq \mathrm{a}$. Then

$$
\int_{E-F} g=R \int_{0}^{\infty} \mu_{g}^{E-F} \leq R \int_{0}^{a} \mu_{g}^{E}<\epsilon,
$$

Q.E.D.

PROOF OF LEMMA 4. In view of Theorem 7 we need only consider the case $g \geq 0$. Given $\epsilon>0$ there is an a $>0$ such that

$$
R \int_{\mathrm{a}}^{\infty} \mu_{\mathrm{g}}^{\mathrm{F}}<\frac{\epsilon}{2}
$$

If $G \subset F$ we have $\mu_{g}^{G} \leqslant m(G)$ and $\mu_{g}^{G} \leq \mu_{g}^{F}$. Then, if we choose $\delta=\epsilon / 2 a$ and if $\mathrm{m}(\mathrm{G})<\delta$, we obtain

$$
\begin{aligned}
\int_{G} g & =R \int_{0}^{a} \mu_{g}^{G}+R \int_{a}^{\infty} \mu_{g}^{G} \\
& \leq a m(G)+R \int_{a}^{\infty} \mu_{g}^{F} \\
& <a m(G)+\frac{\epsilon}{2}<\epsilon,
\end{aligned}
$$

Q.E.D.

In some applications the sequence $\left\{f_{N}\right\}$ may not be uniformly bounded by an integrable function. Such a case frequently occurs when the $f_{N}$ are the partial sums of a series of positive functions. In this type of situation Theorem 11 below is useful, but 1 order to prove 1 t we need some preparation. First we observe that the Lebesgue integral of a nonnegative function can be characterized in terms of those of bounded integrable functions.

LEMMA 5. Let $E \subset \mathbb{R}^{\mathrm{n}}$ be a measurable set and let $\mathrm{f}: E \rightarrow \mathbb{R}$ be a nonnegative measurable function. Then

$$
\int_{E} f=\sup \int_{E} B
$$

where the supremum is taken over all bounded functions $g \in L(E)$ such that $0 \leq g \leq f$. PROOF. If $f \in L(E)$, given $\epsilon>0$ there is $a b>0$ such that

$$
0 \leq \int_{E} f-R \int_{0}^{b} \mu_{f}<\epsilon .
$$

If we define $g=\min \{f, b\}$ then $g$ is bounded, $0 \leq g \leq f, \mu_{g}(y)=\mu_{f}(y)$ if $0<$ $\mathrm{y}<\mathrm{b}$ and $\mu_{\mathrm{g}}(\mathrm{y})=0$ if $\mathrm{y} \geq \mathrm{b}$. We have

$$
\int_{E} g=R \int_{0}^{b} \mu_{g}=R \int_{0}^{b} \mu_{f},
$$

so that $g \in L(E)$ and

$$
0 \leq \int_{E} f-\int_{E} g<\epsilon .
$$

If $f \notin L(E)$ define $E_{a}=\{x \in E: f(x)>$ a $\}$ for each $a>0$ and consider two cases. 
(1) $m\left(E_{a}\right)<\infty$ for all $a>0$. For any $M>0$ there are $a, b \in \mathbb{R}$ such that $0<a<b$ and

$$
R \int_{a}^{b} \mu_{f}>M
$$

If we define $g: E \rightarrow \mathbb{R}$ by $g=\min \{f, b\}$ on $E_{a}$ and $g=0$ on $E-E_{a}$ then $g$ is bounded, $0 \leq \mathrm{g} \leq \mathrm{f}, \mu_{\mathrm{g}}(\mathrm{y})=\mathrm{m}\left(\mathrm{E}_{\mathrm{a}}\right)$ if $0<\mathrm{y}<\mathrm{a}, \mu_{\mathrm{g}}(\mathrm{y})=\mu_{\mathrm{f}}(\mathrm{y})$ if $a \leq y<b$ and $\mu_{g}(y)=0$ if $y \geq b$. It follows that $g \in L(E)$ and that

$$
\int_{E} \mathbf{g}=R \int_{0}^{a} m\left(E_{a}\right)+R \int_{a}^{b} \mu_{f}>a m\left(E_{a}\right)+M
$$

(2) $m\left(E_{a}\right)=\infty$ for some a $>0$. Given $M>0$ let $R$ be a rectangle so large that $m\left(R \cap E_{a}\right)>M / a$. If we define $g(x)=a$ if $x \in R \cap E_{a}$ and $g(x)=0$ otherwise, then $g$ is bounded, $0 \leq \mathrm{g} \leq \mathrm{f}$ and

$$
\int_{E} \boldsymbol{g}=\int_{R \cap E_{a}} a=a m\left(R \cap E_{a}\right)>M \text {, }
$$

Q.E.D.

THEOREM 10. (Fatou's lemma) Let $E \subset \mathbb{R}^{n}$ be a measurable set, let $\left\{\mathrm{f}_{N}\right\}$ be a sequence of nonnegative measurable functions on $E$ and let $f_{N} \rightarrow f$. Then for any $\epsilon>0$ there is an $M \in \mathbb{Z}^{+}$such that

$$
\int_{E} f<\int_{E} f_{N}+\epsilon
$$

for $N>M$.

PROOF. For any $\epsilon>0$, Lemma 5 implies that there is a bounded function $g \in$ $L(E)$, such that $0 \leq B \leq f$ and

$$
\int_{E} f-\int_{E} g<\frac{\epsilon}{2}
$$

If we define $g_{N}=\min \left\{g, f_{N}\right\}$ for each $N \in \mathbb{Z}^{+}$then $g$ is measurable because $\left\{x \in E: g_{N}(x)>y\right\}=\{x \in E: g(x)>y\} \cap\left\{x \in E: f_{N}(x)>y\right\}$ for each $y>0$, and $g_{N} \leq f_{N}$ implies that

$$
\int_{E} g_{N} \leq \int_{E} f_{N}
$$

Also, since $g_{N} \rightarrow g$ on $E$ and $\left|g_{N}\right| \leq 8$, the Lebesgue dominated convergence theorem implies that

$$
\int_{E} g_{N} \rightarrow \int_{E} g
$$

as $N \rightarrow \infty$. Therefore, there is an $M>0$ such that

$$
\int_{E} 8 \leq \int_{E} f_{N}+\frac{\epsilon}{2}
$$

for $N>M$, or else we would have

$$
\int_{E} g_{N} \leq \int_{E} f_{N}<\int_{E} g-\frac{\epsilon}{2}
$$

for arbitrarily large $N$, contradicting the limit above. We conclude that 


$$
\int_{E} f<\int_{E} g+\frac{\epsilon}{2}<\int_{E} f_{N}+\epsilon
$$

for $N>M$, Q.E.D.

THEOREM 11. (The Levi monotone convergence theorem) Let $E \subset \mathbb{R}^{n}$ be a measurable set, let $\left\{f_{N}\right\}$ be a sequence of nonnegative functions on $E$ such that ${ }_{M} \leq f_{N}$ for $\mathrm{M}<\mathrm{N}$, and let $\mathrm{f}_{\mathrm{N}} \rightarrow \mathrm{f}$. Then

$$
\int_{E} f_{N} \rightarrow \int_{E} f
$$

as $\mathrm{N} \rightarrow \infty$.

PROOF. $\mathrm{f}_{\mathrm{M}} \leq \mathrm{f}_{\mathrm{N}}$ for $\mathrm{M}<\mathrm{N} \Rightarrow \mathrm{f}_{\mathrm{N}} \leq \mathrm{f}$ for all $\mathrm{N} \in \mathbb{Z}^{+} \Rightarrow$

$$
\int_{E} f_{N} \leq \int_{E} f
$$

for all $N \in \mathbb{Z}^{+}$. By Fatou's lemma, given $\epsilon>0$

$$
\int_{E} f<\int_{E} f_{N}+\epsilon
$$

for $\mathrm{N}$ large enough. These two inequalities imply that

$$
\int_{E} f_{N} \rightarrow \int_{E} f
$$

as $N \rightarrow \infty$, Q.E.D.

\section{REFERENCES}

1. ROYDEN, H.L. Real Analysis, 2nd. ed., Macmillan, New York, 1968.

2. APOSTOL, T.M. Mathematical Analysis, 2nd. ed., Addison-Wesley, Reading, Mass., 1974 .

3. MCSHANE, E.J. Unified Integration, Academic Press, Orlando, Fla., 1983.

4. MIKUSINSKI, J. The Bochner Integral, Birkhäuser Verlag, Boston, 1978.

5. REY PASTOF, J. Elementos de la Teoría de Funciones, 3rd. ed., Ibero-Americana, Madrid, 1953.

6. KESTELMAN, H. Modern Theories of Integration, Oxford University Press, Oxford, 1937. 


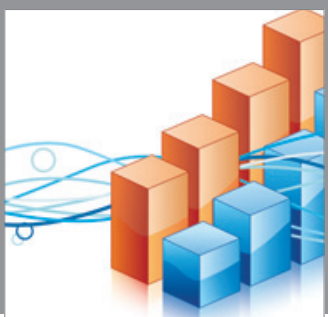

Advances in

Operations Research

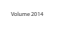

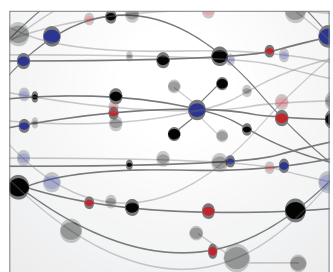

\section{The Scientific} World Journal
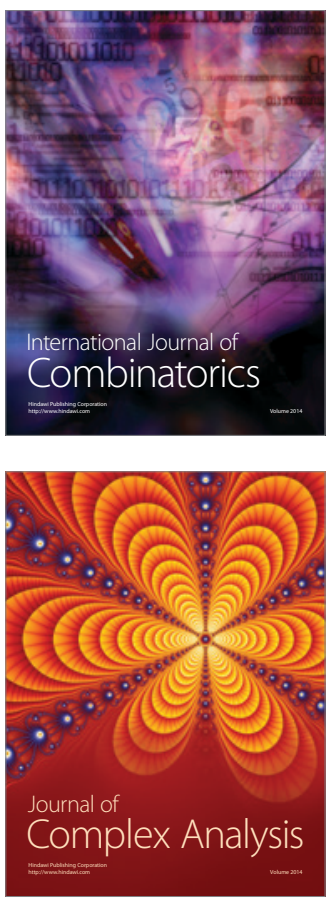

International Journal of

Mathematics and

Mathematical

Sciences
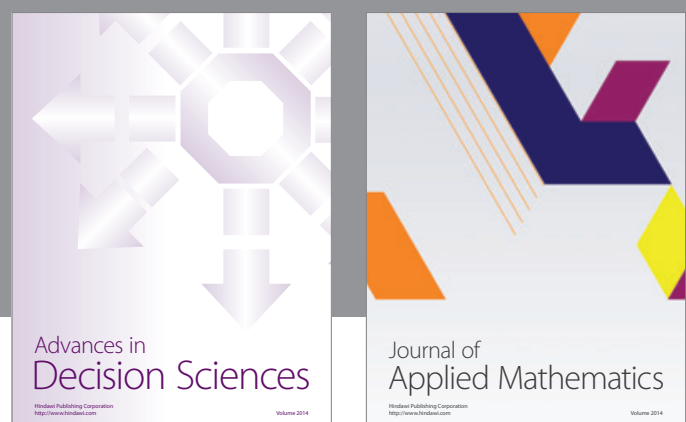

Journal of

Applied Mathematics
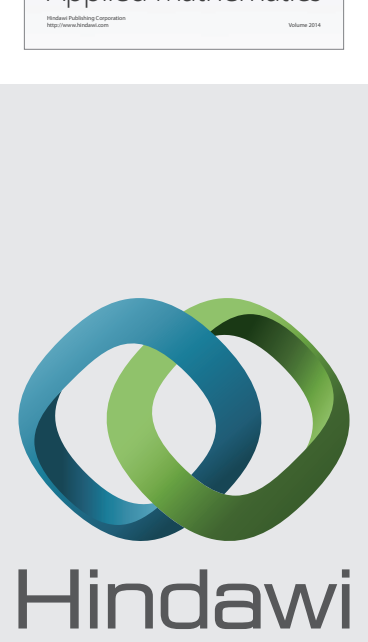

Submit your manuscripts at http://www.hindawi.com
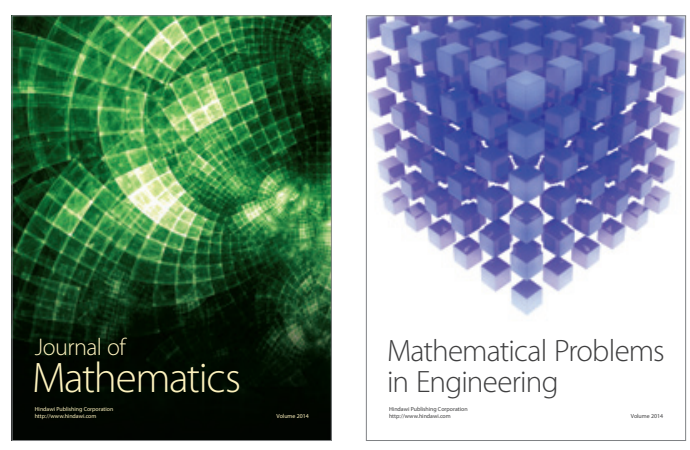

Mathematical Problems in Engineering
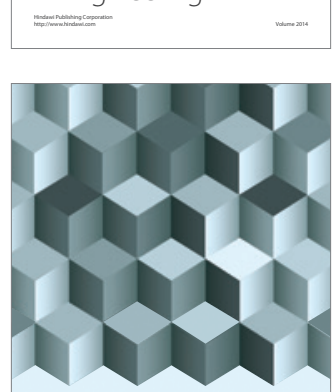

Journal of

Function Spaces
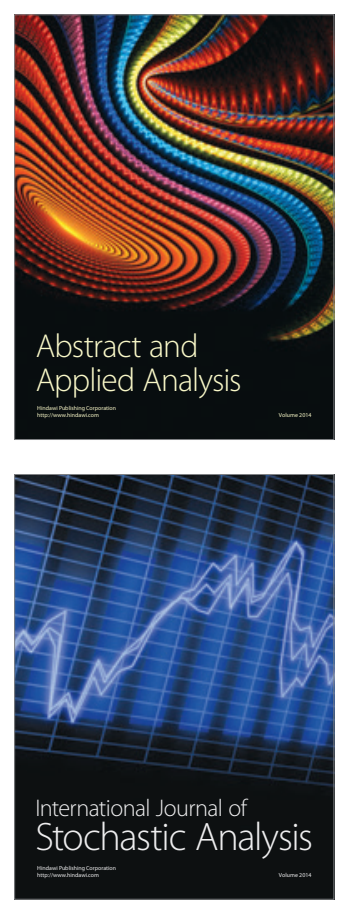

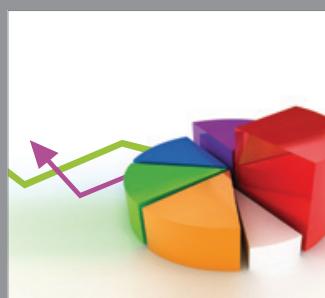

ournal of

Probability and Statistics

Promensencen
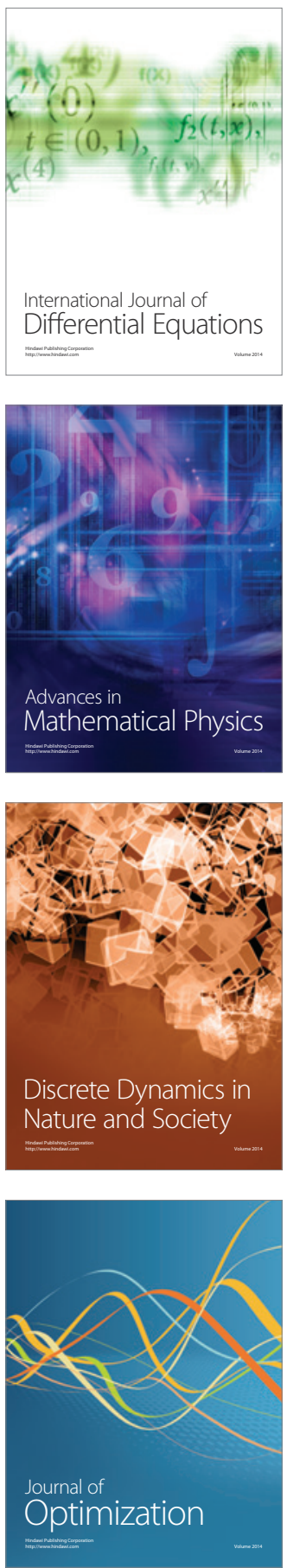